\title{
The effect of nebulized salbutamol on atrial electrical properties in mechanically ventilated critically ill patients - a randomized, double-blind study
}

\author{
Tomasz Jasiński ${ }^{1}$, Radosław Owczuk ${ }^{2}$, Magdalena Wujtewicz ${ }^{2}$ \\ ${ }^{1}$ Department of Anaesthesiology and Intensive Therapy, University Clinical Centre in Gdańsk, Poland \\ ${ }^{2}$ Department of Anaesthesiology and Intensive Therapy, Faculty of Medicine, Medical University \\ of Gdańsk, Poland
}

\begin{abstract}
Background: Salbutamol is a short acting beta- 2 mimetic commonly used among intensive care unit patients. There are data suggesting that his mechanism of action can be a potential factor triggering arrhythmias. The aim of this study was to assess whether nebulized salbutamol causes systemic effects resulting in electrocardiographic alterations associated with atrial fibrillation occurrence in mechanically ventilated patients.

Methods: 50 individuals were randomly allocated to receive either 2.5 or $5 \mathrm{mg}$ of nebulized salbutamol for 20 minutes. 60 minutes prior to the start of nebulization, 12 lead surface electrocardiogram has been recorded. Electrocardiographic parameters associated with atrial activity - P wave and PR interval, and their derivates: $\mathrm{P}$ wave dispersion and PR interval dispersion were analysed.

Results: Both doses of inhaled salbutamol caused changes of $\mathrm{P}$ wave indices - maximal, minimal and mean $\mathrm{P}$ wave duration, from simultaneously recorded leads, and concomitant decrease of $\mathrm{P}$ wave dispersion. Prolongation of $\mathrm{P}$ wave affected predominantly minimal $\mathrm{P}$ wave duration (11.93 ms [95\% Cl: 8.69-15.17]) comparing with maximal $\mathrm{P}$ wave duration (2.61 [95\% Cl: 021-5.43]). Atrio-ventricular conduction — PR interval was also prolonged, however minimal PR interval duration from simultaneously recorded leads was shortened in group receiving $5 \mathrm{mg}$ of salbutamol. Conclusions: Nebulized salbutamol in ICU patient possess systemic effects which causes changes in $P$ wave indices in surface ECG recordings. This electrophysiological influence may be associated with the increased risk of atrial fibrillation among this group of patients.
\end{abstract}

Anaesthesiology Intensive Therapy 2018, vol. 50, no 4, 270-276

Key words: bronchoconstriction, pharmacotherapy, salbutamol; bronchodilator agents, salbutamol; intensive care; mechanical ventilation; atrial fibrillation

Study registered at ClinicalTrials.gov, Trial Registry Number NCT01714401

Atrial fibrillation (AF) is a very common type of arrhythmia among intensive care units (ICU) patients. It is characterised by uncoordinated atrial activation with consequent deterioration of atrial mechanical function. Its frequency varies from 5.8 [1] to $15 \%$ [2] or even $61.7 \%$ [3] depending on the ICU profile: medical or surgical which is many times higher than general population where the frequency is $2 \%$ [4]. The reason for that is the difference between general popu- lation and the population of critically ill. This group presents common risk factors for AF such as older age, hypertension, ischaemic heart disease, rheumatic heart disease, hyperthyroidism, cardiomyopathy, mitral valve pathology, haemochromatosis and infection [5] as well as specific triggers such as anaemia, systemic inflammation, hypoxia, electrolyte imbalances, pain, the use of catecholamines [6]. This arrhythmia is associated with a prolonged ICU duration [7] and 
increased mortality among critically ill [8]; however, there are studies suggesting that $A F$ is rather a marker of disease severity without major impact on mortality [9].

It is also known that drugs can be a triggering factor for AF by means of mechanisms like sympathetic or parasympathetic stimulation, direct cardiotoxicity, alterations in cardiac conduction, refraction and atrial automaticity, induction of myocardial ischaemia or by causing electrolyte abnormalities [10]. Sparse data are known about this mechanism of triggering arrhythmias among ICU patients despite the fact that polypharmacy is common among hospitalized patients [11].

One of the drugs commonly used in the ICU is a short acting beta- 2 mimetic salbutamol. There are data suggesting that his mechanism of action - stimulation of beta-2 adrenergic receptors which are also present on myocardial cells can be a potential factor triggering arrhythmias. The importance of this fact is stressed by the observation that inhalational administration of this drug, which should theoretically reduce its systemic effects [12], is associated electrophysiological influence on myocardial cells [13]. Data about clinical significance of this finding are conflicting stating that beta mimetics can increase the risk of cardiac morbidity among patients with chronic obstructive pulmonary disease [14] as well as that there is no influence of bronchodilatory treatment among critically ill on heart rate (HR) and incidence of arrhythmias [15]. It is also noticeable that most of the studies about this topic do not cover the ICU population. Therefore, the aim of this study was to verify whether nebulised salbutamol causes systemic beta2 adrenergic receptors stimulation, which manifests through changes in P wave indices of the surface ECG recordings, that may be associated with atrial fibrillation occurrence among mechanically ventilated ICU patients.

\section{METHODS}

The study was approved by the Independent Bioethics Committee for Research Studies at the Medical University of Gdansk. Because all participants of the study were unconscious mechanically ventilated patients and salbutamol nebulisation was a therapeutic intervention based directly on patient's medical condition the conductors of the study were allowed by the Independent Bioethics Committee for Research Studies at the Medical University of Gdańsk not to obtain written, informed consent. 50 people were recruited for this randomized double-blind study trial (registered at ClinicalTrials.gov, Trial Registry Number NCT01714401). The main criterion for inclusion was the presence of clinical features of bronchospasm that required administration of nebulised short acting beta- 2 mimetic. All participants were above 18 years of age. Those with persistent atrial fibrillation were excluded from the study, as well as those with history of ventricular tachyarrhythmia (ventricular fibrillation, ventricular tachycardia torsade de pointes). In the pre-study assessment all patients were investigated for the presence of electrolytic imbalances (serum magnesium $<0.7 \mathrm{mmol}$ $\mathrm{L}^{-1}$, ionized calcium $<1.1 \mathrm{mmol} \mathrm{L}^{-1}$, serum potassium $<3.5$ $\mathrm{mmol} \mathrm{L}^{-1}$ ). If any of the serum electrolyte levels was incorrect its normalization after appropriate treatment was verified by another measurement. Patients with persisting electrolytic imbalances were excluded from the study.

All patients were randomly allocated into two equal groups: a group that was to receive the dose of $2.5 \mathrm{mg}$ of nebulised salbutamol ( 2.5 group, $n=25$ ), the most effective dose for mechanically ventilated patients [1], and a group that was to receive the dose of $5 \mathrm{mg}$ of nebulised salbutamol (S 5 group, $\mathrm{n}=25$ ), the highest single dose according to the product characteristics (available at http://www. http:// www.ca.gsk.com/). A randomization scheme was generated using the generator of Wichmann \& Hill, as modified by McLeod (available at http://www.randomization.com).

All patients qualified for the study were assessed according to the APACHE II severity score classification system (available at http://www.sfar.org).

15 minutes before salbutamol administration 12-lead Holter system, Mortara $\mathrm{H} 12+$, with the sampling frequency of $1000 \mathrm{~s}^{-1}$, by Mortara Instruments (Milwaukee, USA) were placed and recording commenced. During the conduction of the study routine monitoring for the ICU including ECG, pulse oximetry and invasive arterial blood pressure was continued in order to detect any possible disturbances caused by chronotropic and arrhythmogenic effect of salbutamol. 15 minutes after initiation of Holter system, ECG signals were checked for the quality of recording. After confirmation of the recording second stage of the study was initiated. Salbutamol ( $2.5 \mathrm{mg}$ or $5 \mathrm{mg}$ ) was administered by the membrane nebulizer Aeroneb pro (Aerogen, Galway, Ireland) attached to the Hamilton G5 ventilator (Hamilton Medical AG, Bonaduz, Switzerland). Nebulizer was filled with previously prepared salbutamol solution constituted from appropriate amount of Ventolin $2 \mathrm{mg}$ $\mathrm{mL}^{-1}$ (GlaxoSmthKline, Great Britain) filled to the volume of $4 \mathrm{~mL}$ by $0,9 \% \mathrm{NaCl}$ from the syringe marked with the randomization number. Both the staffmembers, who administered salbutamol, and patients were blinded to the dose administered. The staff member who prepared the solution and knew its composition was permanently and directly available by phone but did not participate during any stage of the study.

Duration of nebulisation was set for 20 minutes according to information about the size of aerosol particles generated by its specification and data about correlation between nebulization time and appropriate nebulization [16]. Holter ECG data were recorded for 60 minutes from initiation of nebuliser.

The acquired Holter ECG data were analysed at 10 time points: before salbutamol administration (" 0 ") and 5, 10, 15, 20, $25,30,40,50$ and 60 minutes following initiation of nebulisation. All ECG parameters' values were read after a 4-fold magnification of the waveform on a monitor with $1920 \times 1080$ reso- 


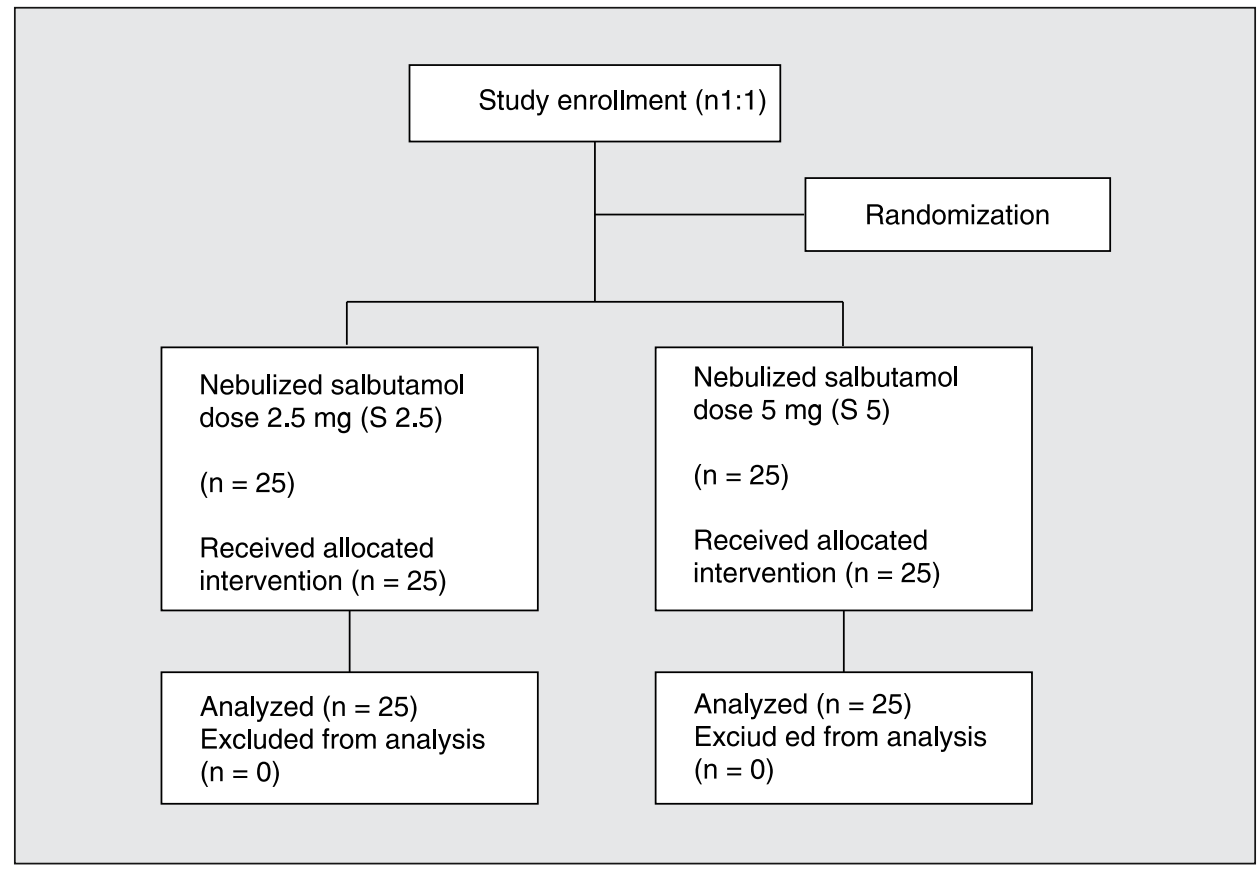

Figure 1. A CONSORT diagram of patient flow in the randomized trial

lution using the Mortara H-scribe 4.01 (Mortara Instruments, Milwaukee, USA). The achieved values are a mean value of five consecutive ECG evolutions. The ECG analysis was performed without knowledge of salbutamol dose used. The results were decoded after completing all electrocardiographic calculations.

In the study two groups of ECG parameters were analysed. The first consisting parameters describing $P$ wave: maximal (Pmax), minimal (Pmin) and mean (Pmean) $P$ wave duration from 12 lead ECG recording at a given time point; $P$ wave dispersion (PWD) - the difference between the longest and the shortest $P$ wave duration from 12 lead ECG recording at a given time point. The second group were ECG parameters describing PR interval: maximal (PRmax), minimal (PRmin) PR interval duration from 12 lead ECG recording at a given time point and PR interval dispersion (PRD) — the difference between the longest and the shortest PR interval duration from 12 lead ECG recording at a given time point. Heart rate (HR) per minute from all time points was also analysed as this parameter can describe sympathomimetic effect of the drug.

A minimal sample size of 18 persons per group was calculated assuming an a level $=0.05$ and $\mathrm{a}$ level $=0.90$ for the detection of a mean difference of $11 \mathrm{~ms}$ considering standard deviation of $P$ wave in population as $10 \mathrm{~ms}$ [15]. In case of possible dropouts, 25 individuals were analysed per group. All data are presented as the mean values $(95 \% \mathrm{Cl})$ or $n$ (proportion). Statistical analysis was performed by using STATISTICA 10.0 PL software (Polish version) (StatSoft, Tulsa, OK, USA). A normal distribution of continuous data was verified with the Shapiro-Wilk's test. Inter and intra group comparisons were performed using two-sided ANOVA tests for repeated measurements after the homogeneity of variance was verified using Levene's test. Subsequent analysis of significant differences was performed using a post hoc method (Tukey HSD test) if appropriate or chi-squared test (with the Yates correction if necessary). $P<0.05$ was adopted as significant.

\section{RESULTS}

A CONSORT diagram depicting patients allocation is shown in Figure 1. Fifty ICU patients undergoing mechanical ventilation were enrolled for this study and were randomly assigned into the two treatment groups. Twenty-five were allocated to the $S 2.5$ group and the $S 5$ group, respectively. All recruited patients completed the study.

Demographic data of patients from both study groups are presented in Table 1. The underlying cause of ICU admission for all enrolled patients was: pulmonary cause $-42 \%$, trauma $-20 \%$, cardiac cause $-14 \%$, surgical cause $10 \%$, neurological cause $8 \%$ and sepsis $6 \%$.

Table 1. Patient details

\begin{tabular}{lcc}
\hline Parameter & $\begin{array}{c}\text { S 2,5 } \\
\mathbf{n}=\mathbf{2 5}\end{array}$ & $\begin{array}{c}\text { S 5 } \\
\mathbf{n}=25\end{array}$ \\
\hline Age (years) & $59(53-65)$ & $57(50-65)$ \\
Apache II score & 27 & 23 \\
Serum potassium (mmol L & & \\
Serum magnesium $\left(\mathrm{mmol} \mathrm{L}^{-1}\right)$ & $0.97(0.93-1.02)$ & $0.98(0.94-1.04)$ \\
lonized calcium $\left(\mathrm{mmol} \mathrm{L}^{-1}\right)$ & $1.14(1.12-1.16)$ & $1.14(1.12-1.18)$ \\
\hline
\end{tabular}




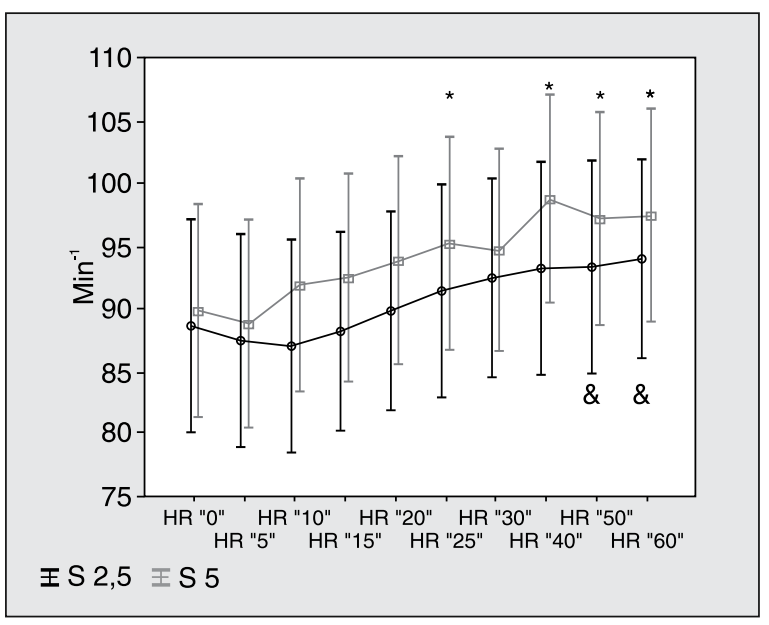

Figure 2. Mean and $95 \% \mathrm{Cls}$ of heart rate per minute $(\mathrm{HR})$ during consecutive time points. " $0^{\prime \prime \prime}, 5^{\prime \prime \prime}, 10^{\prime \prime \prime}, 15^{\prime \prime \prime}, 20^{\prime \prime \prime}, 25^{\prime \prime \prime}, 30^{\prime \prime \prime}, 40$ ','50','60 refer to the time in minutes from the nebulization's beginning. ${ }^{*} P<0.05$ compared with the Initial value in the $S 2.5$ group; ${ }^{\&} P<0.05$ compared with the Initial value in the $S 5$ group

Changes in heart rate (HR) per minute are shown in Figure 2. There was a significant increase in HR during the study in both groups. There were no intergroup differences.
Figures 3(A), 3(B) and 3(C) present changes in the Pmax, Pmin and Pmean in the study groups respectively. There were no intergroup differences in Pmax duration; however, statistical analysis revealed significant intra group Pmax prolongation in the $40^{\text {th }}$ minute of the study comparing with $10^{\text {th }}, 15^{\text {th }}$ and $20^{\text {th }}$ minute of nebulization in the $S 5$ group. No intergroup differences in Pmin duration were found; however, in statistical analysis Pmin prolongation between the initial value comparing with several subsequent measurements were detected in both groups. Statistical analysis revealed Pmean prolongation in both groups.

There were no intergroup differences, but statistical analysis revealed significant PWD reduction between the initial PWD value and subsequent values obtained in both groups as illustrated on Figure 3 (D).

Figures 4 and 5 present changes in the PRmax, PRmin and PRD in the study groups respectively. There were no intergroup differences in PRmax duration, however statistical analysis revealed significant intra group PRmax prolongation between the initial and subsequent measurements in both study groups. No intergroup differences in PRmin duration were found; however, in statistical analysis

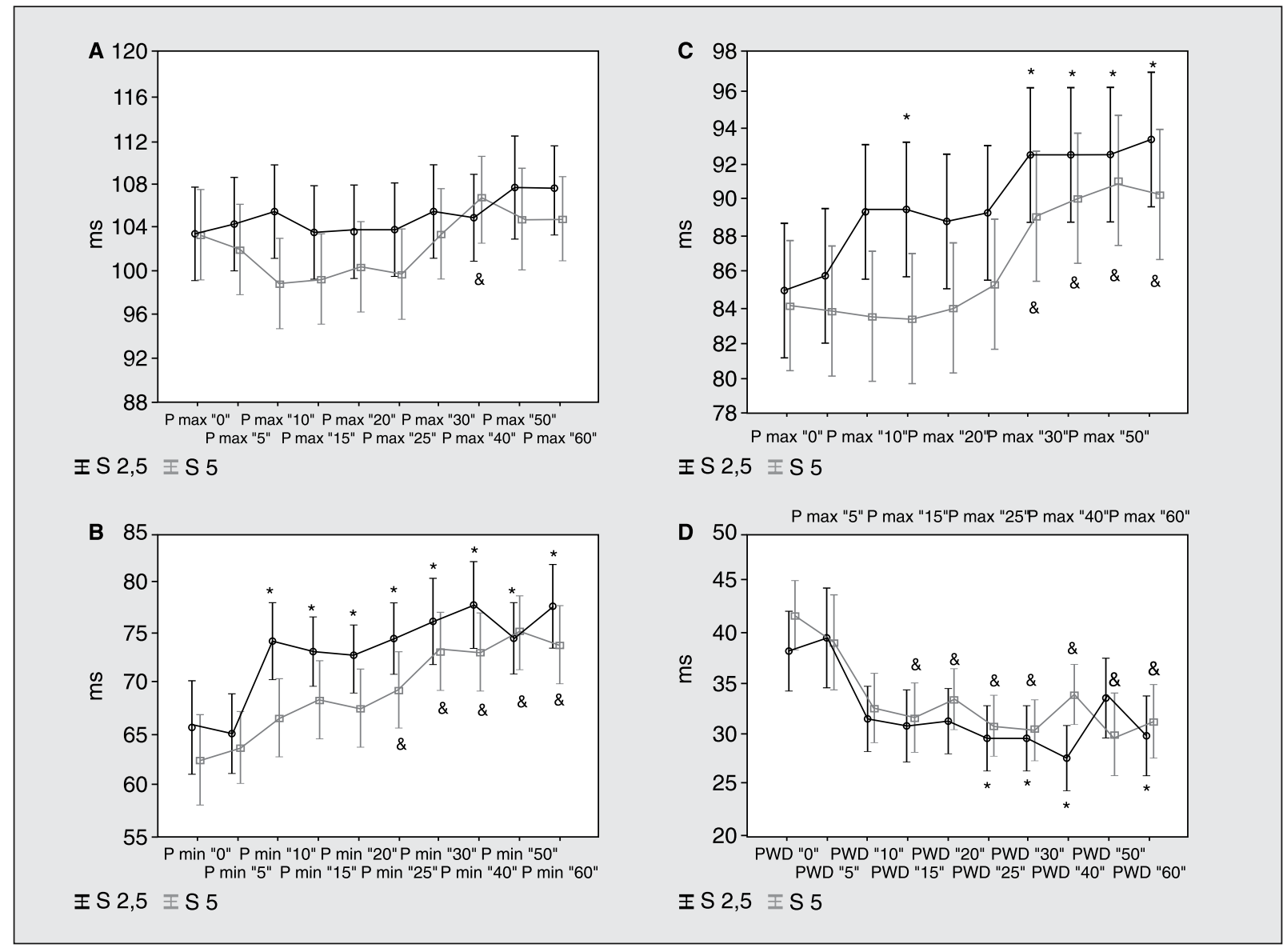

Figure 3. Mean and $95 \% \mathrm{Cls}$ of $(\mathrm{A})$ maximal $\mathrm{P}$ wave duration (Pmax), (B) minimal $\mathrm{P}$ wave duration (Pmin), (C) mean $\mathrm{P}$ wave duration (Pmean), and (D) maximum $P$ wave dispersion (PWD) during consecutive time points. " $0^{\prime \prime \prime \prime}, 5^{\prime \prime \prime}, 10^{\prime \prime \prime}, 15^{\prime \prime \prime \prime}, 20^{\prime \prime \prime}, 25^{\prime \prime \prime \prime}, 30^{\prime \prime \prime}, 40^{\prime \prime \prime}, 50$ ',', 60 refer to the time in minutes from the nebulization's beginning. ${ }^{\&} P<0.05$ compared with the values from initial time points in the $S 5$ group; ${ }^{*} P<0.05$ in the $S 2.5$ group 


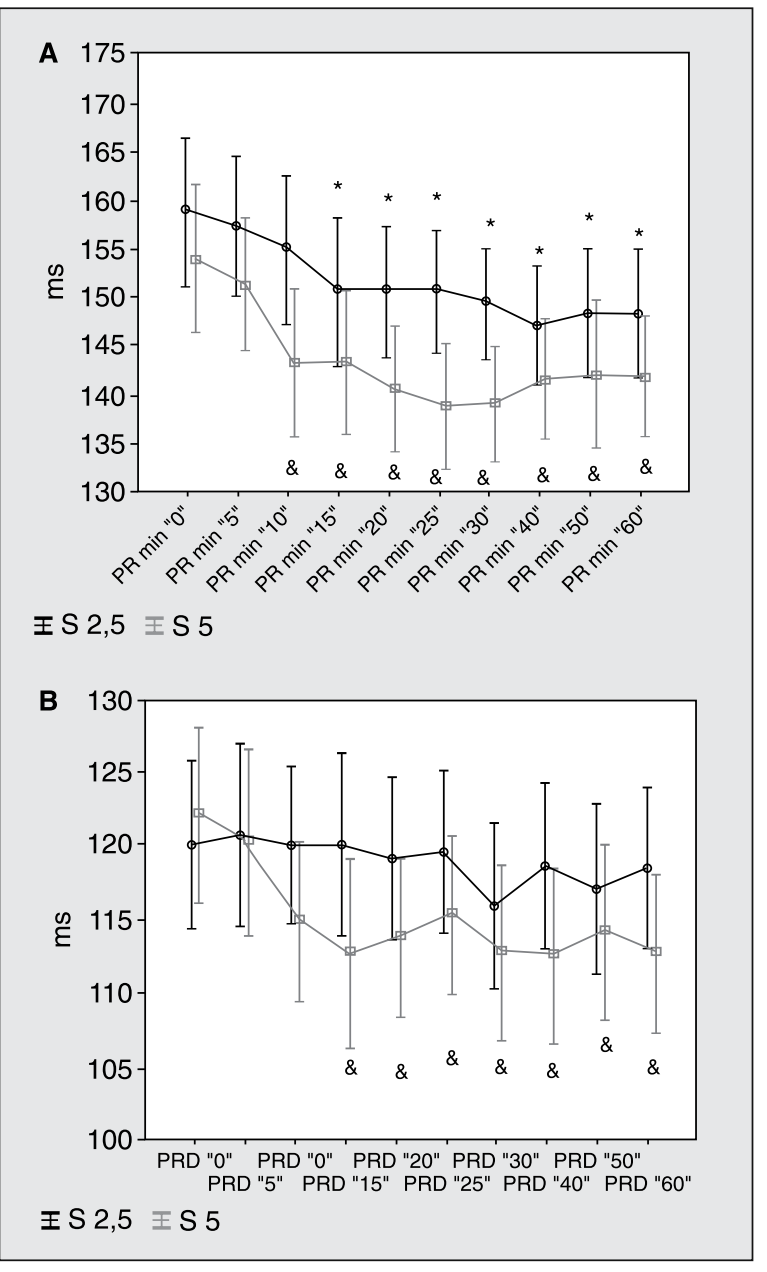

Figure 4. Mean and $95 \% \mathrm{Cls}$ of $(\mathrm{A})$ maximal $\mathrm{PR}$ interval duration (PRmax) (B) and minimal PR interval duration (PRmin) during

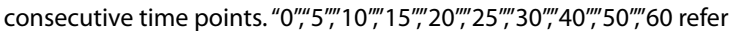
to the time in minutes from the nebulization's beginning. ${ }^{*} P<0.05$ compared with the Initial value in the $S 2.5$ group; ${ }^{\&} P<0.05$ compared with the Initial value in the $S 5$ group

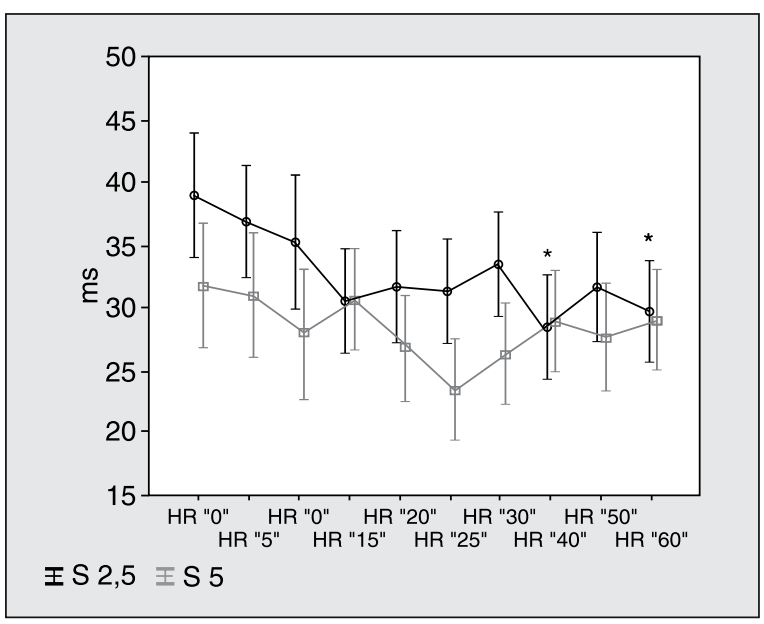

Figure 5. Mean and $95 \% \mathrm{Cls}$ of PR dispersion (PRD) during consecutive time points. " $0^{\prime \prime \prime}, 5^{\prime \prime \prime \prime}, 10^{\prime \prime \prime \prime}, 15^{\prime \prime \prime}, 20^{\prime \prime \prime}, 25^{\prime \prime \prime \prime}, 30^{\prime \prime \prime}, 40^{\prime \prime \prime \prime}, 50^{\prime \prime \prime}, 60$ refer to the time in minutes from the nebulization's beginning. ${ }^{*} P<0.05$ compared with the Initial value in the $S 2.5$ group
PRmin shortening between the initial value and subsequent measurements in the $\mathbf{S} 5$ group was detected. There were no intergroup differences in PRD, but statistical analysis revealed significant $P R D$ reduction between the initial $P R D$ value and the value obtained in $40^{\text {th }}$ and $60^{\text {th }}$ minute of the study in the $S 2,5$ group.

\section{DISCUSSION}

The main finding of our study was that nebulized salbutamol in clinically used doses poses systemic effect in mechanically ventilated critically ill despite the fact that inhalational route of administration should cause this effect neglectable. Clinically it can be observed as an increase in HR which was registered throughout whole study. This effect caused by sympathetic stimulation can be an important factor triggering AF in an ICU population [17].

Apart from the previous observation nebulized salbutamol causes changes in the morphology of surface ECG recordings associated with atrial activity $-P$ wave. From the previous studies it is known that those ECG indices are not only the effect of different projections of the $P$ wave vector which are caused by different angles between the vector and the lead axis [18] but can differ in length between the simultaneously registered leads because of the microarchitecture and the anisotropic properties of atrial myocardium $[19,20]$. Changes in $P$ wave indices are associated with supraventricular arrhythmias like AF [21] although they have a limited contribution toward predictive risk beyond traditional risk factors and markers [22]. In our study, nebulized salbutamol lead to prolongation of all parameters describing $\mathrm{P}$ vawe-Pmax, $\mathrm{P}$ min, Pmean. Studies like Mangani et al. [23] and Gonna et al. [24] suggest that $P$ wave prolongation increase the risk of AF. What seems worth noticing is that increase in Pmin duration $(11.93 \mathrm{~ms}$ [95\% Cl: 8.69-15.17]) exceeds changes in Pmax (2.61 [95\% Cl: 0.21-5.43])].

The observation that may be considered as conflicting or difficult in interpretation, comparing with data published by other authors, is that salbutamol caused PWD decrease throughout the study course [25]. PWD calculated from simultaneously obtained Pmax and Pmin can be increased as an effect of sympathetic stimulation which leads to changes in intra-atrial conduction [26]. This fact suggests that after administration of beta 2 mimetics PWD should increase; however, there is lack of scientific data confirming this hypothesis. PWD increase, especially above $40 \mathrm{~s} 3$, as it is known from other trials, is a factor that may be associated with the risk of AF occurrence [26] and medical interventions [27-29] and administered drugs [30] can have influence on this parameter. The study by Mangani et al. [24] however, which assessed the Framingham Heart Study population failed to detect any significant correlation between PWD and AF. In 
our study salbutamol administered in ICU patients lead to PWD decrease through its course which can be partially explained by, as mentioned before, predominant increase in Pmin duration. It is also crucial that most trials asses the influence of different interventions on PWD in long term observations, not the immediate effect like in our study. This would suggest low predictive value of PWD in the population of critically ill.

The second group of surface ECG parameters examined in the study describe the PR interval and provide information about atrio-ventricular conduction. Analyses of huge populations performed by Cheng et al. [31] and Magnani et al. [32] have showed that PR interval prolongation is strongly correlated with the occurrence of AF. In our study, nebulized salbutamol caused PRmax prolongation which may confirm its AF triggering properties. The data obtained from other PR indices are however difficult to interpret as PRmin duration and PRD value were unaffected or decreased depending on the salbutamol dose. It is important to mention that the value of those two PR indices and their association with arrhythmogenicity have not been examined yet. Also, the data about factors and drugs causing alterations in those two parameters describing atrio-ventricular conduction are sparse.

In conclusion, nebulized salbutamol used in mechanically ventilated ICU patients induced prolongation of atrial depolarization and atrio-ventricular conduction which manifested as prolongation of the $P$ wave and $P R$ interval in the surface 12 lead ECG recording. This effect does not seem to be dose dependent as there were no intragroup differences between two administered doses. As there are convincing evidence from other trials that prolongation of those two indices is associated with episodes of $\mathrm{AF}$ this finding allowed us to conclude that salbutamol possess pro- arythmogenic properties which may be responsible for triggering atrial fibrillation in this group of patients. As episodes of AF among ICU patents have multifactorial origin further studies are required to assess the clinical significance of those findings.

\section{ACKNOWLEDGEMENTS}

1. This study was supported by Medical University of Gdansk (grant number ST - 64).

2. Conflict of interest: none.

\section{References:}

1. Meierhenrich R, Steinhilber E, Eggermann C, et al. Incidence and prognostic impact of new-onset atrial fibrillation in patients with septic shock: a prospective observational study. Crit Care. 2010; 14(3): R108, doi: 10.1186/cc9057, indexed in Pubmed: 20537138.

2. Reinelt P, Karth GD, Geppert A, et al. Incidence and type of cardiac arrhythmias in critically ill patients: a single center experience in a medical-cardiological ICU. Intensive Care Med. 2001; 27(9): 1466-1473, doi: 10.1007/s001340101043, indexed in Pubmed: 11685339.
3. Puentes W, Jerath A, Djaiani G, et al. Early versus late tracheostomy in cardiovascular intensive care patients. Anaesthesiol Intensive Ther. 2016; 48(2): 89-94, doi: 10.5603/AIT.a2016.0016, indexed in Pubmed: 26977852

4. Lloyd-Jones DM, Wang TJ, Leip EP, et al. Lifetime risk for development of atrial fibrillation: the Framingham Heart Study. Circulation. 2004; 110(9): 1042-1046, doi: 10.1161/01.CIR.0000140263.20897.42, indexed in Pubmed: 15313941.

5. Cavaliere F, Volpe $C$, Soave M. Atrial fibrillation in intensive care units. Current Anaesthesia \& Critical Care. 2006; 17(6): 367-374, doi: 10.1016/j. cacc.2006.12.002.

6. Yoshida T, Fujii T, Uchino S, et al. Epidemiology, prevention, and treatment of new-onset atrial fibrillation in critically ill: a systematic review. J Intensive Care. 2015;3(1): 19, doi: 10.1186/s40560-015-0085-4, indexed in Pubmed: 25914828.

7. Koyfman L, Brotfain E, Kutz R, et al. Epidemiology of new-onset paroxysmal atrial fibrillation in the General Intensive Care Unit population and after discharge from ICU. A retrospective epidemiological study. Anaesthesiol Intensive Ther. 2015; 47(4): 309-314, doi: 10.5603/AIT. a2015.0040, indexed in Pubmed: 26210522.

8. Brathwaite D, Weissman C. The new onset of atrial arrhythmias following major noncardiothoracic surgery is associated with increased mortality. Chest. 1998; 114(2): 462-468, indexed in Pubmed: 9726731.

9. Seguin $P$, Launey Y. Atrial fibrillation is not just an artefact in the ICU. Crit Care. 2010; 14(4): 182, doi: 10.1186/cc9093, indexed in Pubmed: 20670386.

10. van der Hooft CS, Heeringa J, van Herpen G, et al. Drug-induced atrial fibrillation. J Am Coll Cardiol. 2004; 44(11): 2117-2124, doi: 10.1016/j. jacc.2004.08.053, indexed in Pubmed: 15582307.

11. Trumic E, Pranjic N, Begic L, et al. Prevalence of polypharmacy and drug interaction among hospitalized patients: opportunities and responsabilities in pharmaceutical care. Mater Sociomed. 2012; 24(2): 68-72, doi: 10.5455/msm.2012.24.68-72, indexed in Pubmed: 23678310.

12. Duarte AG, Dhand R, Reid R, et al. Serum albuterol levels in mechanically ventilated patients and healthy subjects after metered-dose inhaler administration. Am J Respir Crit Care Med. 1996; 154(6 Pt 1): 1658-1663, doi: 10.1164/ajrccm.154.6.8970351, indexed in Pubmed: 8970351.

13. Kallergis EM, Manios EG, Kanoupakis EM, et al. Acute electrophysiologic effects of inhaled salbutamol in humans. Chest. 2005; 127(6): 20572063, doi: 10.1378/chest.127.6.2057, indexed in Pubmed: 15947320.

14. Salpeter SR, Buckley NS, Ormiston TM, et al. Meta-analysis: effect of long-acting beta-agonists on severe asthma exacerbations and asthma-related deaths. Ann Intern Med. 2006; 144(12): 904-912, indexed in Pubmed: 16754916.

15. Khorfan FM, Smith P, Watt S, et al. Effects of nebulized bronchodilator therapy on heart rate and arrhythmias in critically ill adult patients. Chest. 2011; 140(6): 1466-1472, doi: 10.1378/chest.11-0525, indexed in Pubmed: 21960699.

16. Duarte AG. Inhaled bronchodilator administration during mechanical ventilation. Respir Care. 2004; 49(6): 623-634, indexed in Pubmed: 15165297.

17. Magnani JW, Williamson MA, Ellinor PT, et al. P wave indices: current status and future directions in epidemiology, clinical, and research applications. Circ Arrhythm Electrophysiol. 2009; 2(1): 72-79, doi: 10.1161/ CIRCEP.108.806828, indexed in Pubmed: 19808445.

18. Michelucci A, Padeletti L, Chelucci A, et al. Influence of age, lead axis, frequency of arrhythmic episodes, and atrial dimensions on $P$ wave triggered SAECG in patients with lone paroxysmal atrial fibrillation. Pacing Clin Electrophysiol. 1996; 19(5): 758-767, indexed in Pubmed: 8734742.

19. Spach MS, Dolber PC. Relating extracellular potentials and their derivatives to anisotropic propagation at a microscopic level in human cardiac muscle. Evidence for electrical uncoupling of side-to-side fiber connections with increasing age. Circ Res. 1986; 58(3): 356-371, indexed in Pubmed: 3719925.

20. Spach MS, Miller WT, Geselowitz DB, et al. The discontinuous nature of propagation in normal canine cardiac muscle. Evidence for recurrent discontinuities of intracellular resistance that affect the membrane currents. Circ Res. 1981; 48(1): 39-54, indexed in Pubmed: 7438345.

21. De Bacquer D, Willekens J, De Backer G. Long-term prognostic value of $p$-wave characteristics for the development of atrial fibrillation in subjects aged 55 to 74 years at baseline. Am J Cardiol. 2007; 100(5): 850-854, doi: 10.1016/j.amjcard.2007.04.017, indexed in Pubmed: 17719332. 
22. Magnani JW, Zhu L, Lopez F, et al. P-wave indices and atrial fibrillation: cross-cohort assessments from the Framingham Heart Study (FHS) and Atherosclerosis Risk in Communities (ARIC) study. Am Heart J. 2015; 169(1): 53-61.e1, doi: 10.1016/j.ahj.2014.10.009, indexed in Pubmed: 25497248.

23. Gonna $\mathrm{H}$, Gallagher MM, Guo XH, et al. P-wave abnormality predicts recurrence of atrial fibrillation after electrical cardioversion: a prospective study. Ann Noninvasive Electrocardiol. 2014; 19(1):57-62, doi: 10.1111/ anec.12087, indexed in Pubmed: 24320565.

24. Magnani JW, Johnson VM, Sullivan LM, et al. P wave duration and risk of longitudinal atrial fibrillation in persons $\geq 60$ years old (from the Framingham Heart Study). Am J Cardiol. 2011; 107(6): 917-921.e1, doi: 10.1016/j.amjcard.2010.10.075, indexed in Pubmed: 21255761.

25. Dilaveris PE, Gialafos EJ, Sideris SK, et al. Simple electrocardiographic markers for the prediction of paroxysmal idiopathic atrial fibrillation. Am Heart J. 1998; 135(5 Pt 1): 733-738, indexed in Pubmed: 9588401.

26. Twardowski P, Owczuk R, Wujtewicz MA, et al. The influence of epidural anesthesia on the electrical activity of heart atria. Kardiochir Torakochirurgia Pol. 2014; 11(2): 156-161, doi: 10.5114/kitp.2014.43843, indexed in Pubmed: 26336414.

27. Materazzo C, Piotti P, Mantovani C, et al. Atrial fibrillation after non-cardiac surgery: P-wave characteristics and Holter monitoring in risk assessment. Eur J Cardiothorac Surg. 2007; 31(5): 812-816, doi: 10.1016/j.ejcts.2007.02.007, indexed in Pubmed: 17337197.

28. Akdemir R, Ozhan $\mathrm{H}$, Gunduz $\mathrm{H}$, et al. Effect of reperfusion on P-wave duration and $\mathrm{P}$-wave dispersion in acute myocardial infarction: primary angioplasty versus thrombolytic therapy. Ann Noninvasive Electrocardiol. 2005; 10(1): 35-40, doi: 10.1111/j.1542-474X.2005.00595.x, indexed in Pubmed: 15649235.
29. Lazzeroni D, Parati G, Bini M, et al. P-wave dispersion predicts atrial fibrillation following cardiac surgery. Int J Cardiol. 2016; 203: 131-133, doi: 10.1016/j.ijcard.2015.10.143, indexed in Pubmed: 26512825.

30. Celik T, lyisoy A, Kursaklioglu $\mathrm{H}$, et al. The comparative effects of telmisartan and ramipril on $\mathrm{P}$-wave dispersion in hypertensive patients: a randomized clinical study. Clin Cardiol. 2005; 28(6): 298-302, indexed in Pubmed: 16028466.

31. Cheng S, Keyes MJ, Larson MG, et al. Long-term outcomes in individuals with prolonged PR interval or first-degree atrioventricular block. JAMA. 2009; 301(24): 2571-2577, doi: 10.1001/jama.2009.888, indexed in Pubmed: 19549974.

32. Magnani JW, Wang Na, Nelson KP, et al. Health, Aging, and Body Composition Study. Electrocardiographic PR interval and adverse outcomes in older adults: the Health, Aging, and Body Composition study. Circ Arrhythm Electrophysiol. 2013; 6(1): 84-90, doi: 10.1161/ CIRCEP.112.975342, indexed in Pubmed: 23243193.

\section{Corresponding author}

Tomasz Jasiński

Department of Anaesthesiology and Intensive Therapy

University Clinical Centre in Gdańsk

Smoluchowskiego 17, 80-214 Gdańsk, Poland

e-mail:tjasinski@uck.gda.pl

Received: 28.06.2018

Accepted: 18.09.2018 